(11) M. Kawamoto and M. Seki : Jour. Japan Soc. Test. Mat., Vol.5, No.35, (1956), p.486.

(12) S. Hukai : Jour. Japan Soc. Test. Mat., Vol.6, No. 45, (1957), p.397.

(13) A.R. Wade and P. Grootenhuis: Proc. Inter. Conf. on Fatigue of Metals, (1956), p,361.

(14) T.W. Romas, J.O. Ward, J.R. Rait, and E.W. Colbeck : Proc. Inter. Conf. on Fatigue of Metals, (1956), p. 375 .

(15) H.F. Moore and N.J. Allemann : Proc. ASTM, Vol. 31, (1931) p. 114.

(16) Committee E-1, Proc. ASTM, Vol.50,(1950), p. 421.

(17) G.R. Gohn and W.C. Ellis: Proc. ASTM, Vol. 51, (1951), p. 721

(18) J.F. Eckel : Proc. ASTM, Vol.51, (1951), p. 745.

(19) P.G. Forrest and H.J. Tapsell: Engineering, (June$13,1952)$, p. 757

(20) P.G. Forrest and H.J. Tapsel1 : Proc. Inst. Mech.
Engrs., Vol.168, (1954), p. 763

(21) N.P. Allen and P.G. Forrest: Proc. Inter, Conf. on Fatigue of Metals, (1956), p. 227.

(22) J.C. Levy and G.M. Sinclair: Proc. ASTM, Vol.55, (1955), p.866.

(23) M. Kawamoto, T.Ishida, and H. Kakuzen : Jour. Japan Soc. Test. Mat., Vol.5, No.30, (1956), p. 161.

(24) S. Taira and R.Koterazawa: Jour. Japan Soc. Test. Mat., Vol.6, No.45, (1957), p.401.

(25) S. Taira and R. Koterazawa:Trans. Japan Soc. Mech. Engrs., Vol. 25, No.159, (1959), p. 1064.

(26) M. Kawamoto; T.Tanaka, and S. Tabuchi : Jour. Japan Soc. Test. Mat., Vol.9, No.87, (1960), p. 742.

(27) M. Kawamoto, T. Tanaka, and M. Miyake: Trans, Japan Soc Mech. Engrs., Vol. 27, No. 176, (1961), p. 403 .

(28) M. Kawamoto and S. Tabuchi : Jour. Japan Soc. Test. Mat., Vol.9, No.82, (1960), p.452.

$539.43: 620.179 .152$

\title{
X-Ray Investigation on the Prediction of Fatigue Life*
}

\author{
(On the Changes in Half-Value Breadth of Carbon \\ Steel under Varying Stress Amplitudes)
}

\author{
By Shuji TAIRA** and Kazuo HondA***
}

It has been reported by the authors that the estimation of fatigue life of the materials under constant stress amplitude can be made using the liner relation of $b / B-\log n / N$ (where $B$ and $b$ are the initial and the current half-value breadth of diffraction line in fatigue and $n / N$ is the cycle ratio).

In the present paper, the following experiments were performed; the first, the experiments were made on the case of varying stress amplitude of two stress levels above fatigue limit and investigated the change of half-value breadth due to varying stress amplitude. The second, the half-value breadth was measured by using satisfactory method in physical meaning and compared with the conventional method which has been adopted in these series of investigations. Finally, discussed on a change of half-value breadth under varying stress amplitude and postulated a certain method for nondestructive estimation of fatigue life.

\section{Introduction}

A reliable means of predicting the fracture of machine and structural members due to fatigue would be of immense importance for machine designers and metallurgists. For many years, using various techniques intensive investigations have been made on this problem, but, the fundamental nature of fatigue being complex and obscure, lack of definite information about it has not yet allowed establishment of a

* Received 28th August, 1961.

** Professor, Faculty of Engineering, Kyoto University.

*** Assistant, Faculty of Engineering, Kyoto University, Sakyo-ku, Kyoto. suitable means which is practical in design.

In this connection, the authors have investigated the fatigue process, for some years by using the $\mathrm{X}$-ray technique and studied the mechanism and also the amount of fatigue damage by observing the variation in the half-value breadths taken from X-ray diffraction lines which were obtained from variously treated carbon steel, cast iron and cast steel subjected to fatigue under constant stress amplitudes ${ }^{(1)(2)}$. From these results, the authors suggested that the relation between the change in the ratio of half-value breadth $b / B$ and the $\log$ of cycle ratio $n / N$ ( $B$ and $b$ are the initial and current half-value breadths and $n$ and $N$ are the numbers of stress cycles at any 
period and at fracture, respectively) is expressed approximately by a straight line in every case of the annealed, the cold worked and the heat treated materials. Based on this relation, the authors postulated a certain method of nondestructive prediction of fatigue life.

However, in order to establish a universal method of prediction of fatigue damage, the following problems must be examined;

1) Under the service conditions, stress amplitude is not steady but fluctuates with time, and so the investigation of the change in half-value breadth under service loads is necessary for practical purposes.

2) The conventional method of measurement of half-value breadth has been used in a series of experiments, because of lack of any other satisfactory methods. However, it is considered that the exact measurement of half-value breadth is one of the most important problems in these investigations.

3) Moreover, it is assumed that the value of half-value breadth would be influenced by the radiation area of $\mathrm{X}$-ray, the secondary voltage and the other few factors. Accordingly, in the present study the authors carried out some experiments on these problems and discussed the results obtained.

\section{Some basic problems on the measurement of half-value breadth of diffraction line}

$2 \cdot 1$ Reexamination of the measurement of

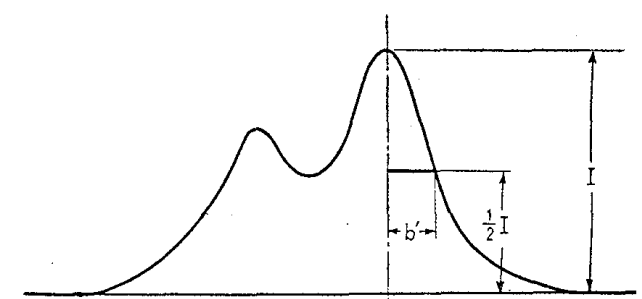

(A) Measurement of half-value breadth of the annealed specimen

\section{half-value breadth}

The studies carried out by the authors previously, aimed at the engineering application of the findings. Thus the convenient method of measurement of halfvalue breadth as shown in Fig. 1 (A, B) was adopted. The reliability of the method for its reproducibility was verified (method 1). However, this mode of measurement of the breadth would not be satisfactory from the physical view point, therefore in the present study, the authors employed a modification of the one that has been proposed by W.A. Wood and Rachinger ${ }^{(3)}$ (method 2). The latter is considered to be more reasonable than any others ${ }^{(4) \sim(7)}$. In the case of measurement of X-ray diffraction line breadth a difficulty is encountered due to the overlop of the $K \alpha_{1}$ and $K \alpha_{2}$ line. The process of the measurement will be described briefly as follows : In Fig. 2(A, B).

a) The distance e between the peaks of $K \alpha_{1}$ and $K \alpha_{2}$ curves is determined by calculation or from the diffraction line of the annealed material.

b) The points $\mathrm{C}$ and $\mathrm{C}^{\prime}$ are so plotted on the base line aa that $\mathrm{AC}$ and $\mathrm{A}^{\prime} \mathrm{C}^{\prime}$ may be equal to the distance e. The $K \alpha_{1}$ curve in the range $\mathrm{AB}$ is considered the true diffraction line which is not influenced by the $K \alpha_{2}$ curve.

c) Plot the middle points $\mathrm{H}_{2}$ and $\mathrm{H}_{2}{ }^{\prime}$ of the lines $A C^{\prime}$ and $A^{\prime} C$, then the vertical lines through these points determine the positions of $K \alpha_{1}$ and $K \alpha_{2}$ peaks.

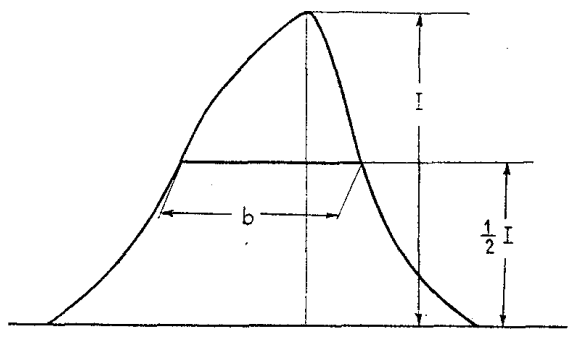

(B) Measurement of half-value breadth of the cold worked specimen

Fig. 1 Conventional method of the measurement of half-value breadth of $X$-ray diffraction line (method 1 )

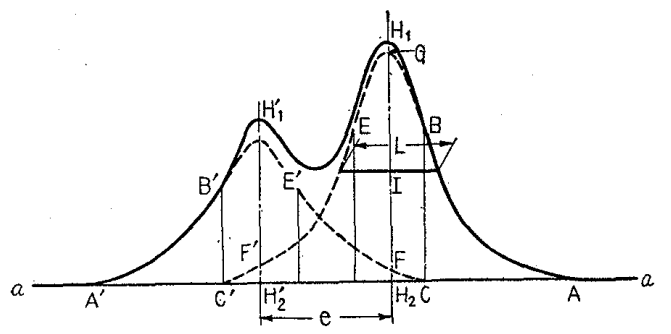

(A) Measurement of half-value breadth of annealed specimen

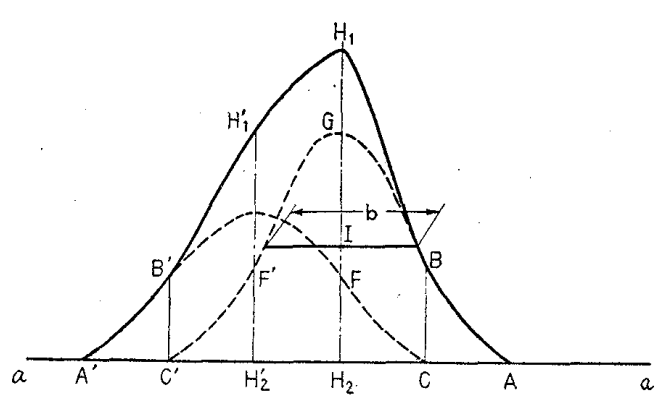

(B) Measurement of half-value breadth of the cold worked specimen

Fig. 2 Modified method of measurement of half-value breadth of X-ray diffraction line (method 2) 
d) The dotted line $E^{\prime} C$ is so drawn as to be symmetrical with $\mathrm{A}^{\prime} \mathrm{B}^{\prime}$ line in regard to $\mathrm{H}_{2}{ }^{\prime} \mathrm{H}_{1}{ }^{\prime}$ line. The value of $\mathrm{FH}_{1}\left(I_{K \alpha_{1}}\right)$ gives the true peak height of the $K \alpha_{1}$ curve.

e) The same procedure is repeated to obtain $F^{\prime} \mathrm{H}_{1}{ }^{\prime}\left(I_{K \alpha_{2}}\right)$.

f) Then, the half-value breadth can be determined from the following relation,

$B=\frac{A\left(\text { area under the } K \alpha_{1} \text { and } K \alpha_{2} \text { diffraction lines }\right)}{I_{K \alpha_{1}}+I_{K \alpha_{2}}}$

To compare this method with the previous one, some experiments were carried out using the annealed and the cold worked wire of $0.42 \%$ carbon steel, which had been subjected to fatigue stressing. $\mathrm{X}$ ray diffractometer of the automatic recording type and photograph method were used in these experiments, and the intensity curves during stress repetitions were obtained as shown in Figs. 3 and 4. From these results, it is seen that the inclination of $b / B-N$ curve is nearly the same in both cases of the methods (1) and (2). Consequently, the conventional method of measurement of the breadth which has been utilized in the series of studies is considered to be satisfactory for the engineering application.

\section{$2 \cdot 2$ Effect of radiation area}

It is considered that the broadened line is an indication of either very small crystallite size or lattice distortion or both. Cold work applied to polygrained metal produces a broadening of the X-ray pattern lines. Most of the earlier discussions have been based either upon the fragmentation theory or upon the micro-stress theory. In the latter theory, it is supposed that residual strains are left in the material, so that they vary in magnitude from one grain (or subgrain) to the next. Each crystal has slightly different lattice spacing, and the reflection beams from various crystals merge into one broadened reflection. From the results of these investigations, it is supposed that the broadening is affected by the area of X-ray radiation. Moreover, recent works in the field of physical metallurgy have pointed out the presence of micro-stresses due to several sorts of lattice defects even in annealed single crystal. From these reasons, it is assumed that the half-value breadth is influenced largely by the radiation area. To study this influence, some experiments were performed, the result being shown in Fig. 5. It is seen that the line broadening is influenced considerably by the radiation area. Therefore, in the measurement of half-value breadth, radiation area should be controlled strictly.

2.3 Effect of the tube voltage and tube current

The X-ray voltage and the current affect on the penetration depth of $\mathrm{X}$-ray and the intensity or

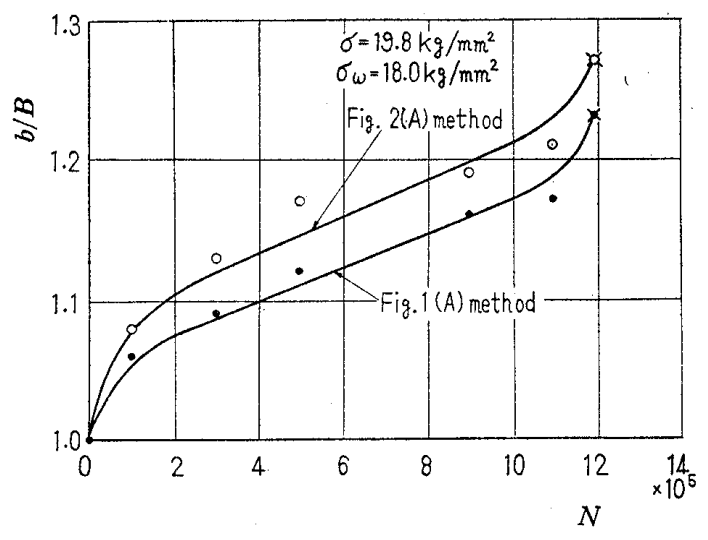

Fig. 3 Changes in half-value breadth due to stress repetitions using two different methods of measurements (annealed specimen)

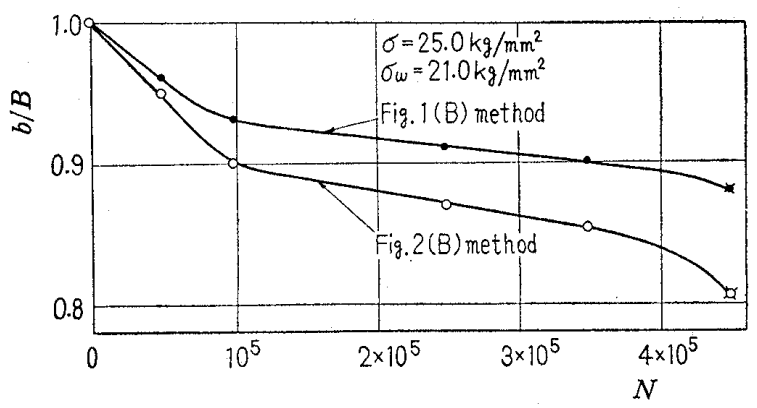

Fig. 4 Changes in half-value breadth due to stress repetitions using two different methods of measurements (cold worked specimen)

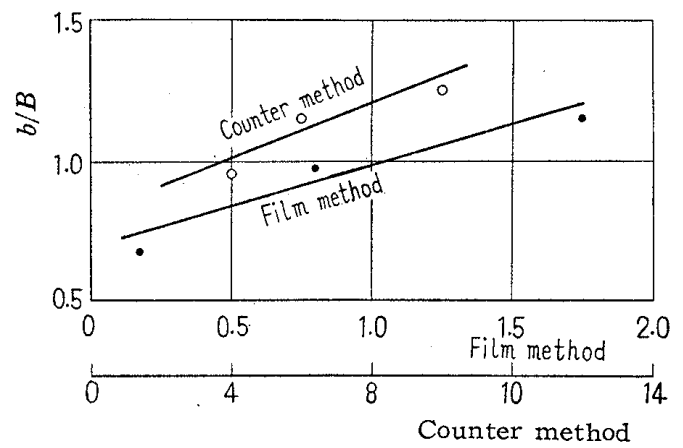

Radiation area $\mathrm{mm}^{2}$

Fig. 5 Effect of radiation area on the value of half-value breadth

the scattering of diffraction line etc ; hence, it is considered that these factors influence the line broadening. Table 1 shows one example of these experimental results. It is to be noted that the variation in the secondary voltage of X-ray and tube current have no effect on the value of halfvalue breadth within experimental errors.

\section{Studies of fatigue under varying stress amplitudes}

As pointed out in the previous section, the investigation of the change in half-value breadth 
under service loads is of vital importance for a practical use, and so some basic experiments under varying stress amplitudes were performed.

\subsection{Specimens and fatigue tests}

Two sorts of materials were tested, the one was an annealed $0.42 \%$ carbon steel wire of $3 \mathrm{~mm}$ dia. and the other a cold drawn wire of the same material. A Shwinning type rotating bending testing machine was used for the fatigue test, and the speed of stress repetitions was 2000 cycles per minute. Table 2 shows the chemical composition of the material, and the endurance limits obtained were 18.0 $\mathrm{kg} / \mathrm{mm}^{2}$ for the annealed specimens and $38.0 \mathrm{~kg} / \mathrm{mm}^{2}$ for the cold worked ones respectively.

Two series of tests were carried out in the present study, one series being the case of ascending or descending stressing in two stress levels, and the other series consisting of multiple repeated stressing in two stress levels above the fatigue limit. Both series of tests were made with the annealed and the cold worked specimens.

The test programs of the first series are shown in Table 3 (A, B), and those of the second series in Table 3 (C). In Table 3 , the magnitudes of the pre-stress $\sigma_{1}$ and the secondary stress $\sigma_{2}$ are chosen both above the fatigue limit of each specimen. $N_{1}$ or $N_{2}$ denotes the number of cycles at fracture when a steady stress amplitude of $\sigma_{1}$ or $\sigma_{2}$ is applied. Specimens were subjected to the pre-stress of $\sigma_{1}$ till $n_{1}$ cycles, and then the stress was increased or decreased to the secondary stress $\sigma_{2}$, with the result that fracture occurred after $n_{2}$ cycles. Thus $n_{1} / N_{1}$ and $n_{2} / N_{2}$ are the cycle ratios under the pre-stress and the secondary stress amplitudes respectively.

Table 1 Effect of X-ray intensity on the value of half-value breadth

\begin{tabular}{l|c|c|c}
\hline \hline X-ray intensity & $25 \mathrm{kV} 10 \mathrm{~mA}$ & $25 \mathrm{kV} 7 \mathrm{~mA}$ & $25 \mathrm{kV} 5 \mathrm{~mA}$ \\
Half-value breadth & $0.95 \mathrm{~mm}$ & $0.97 \mathrm{~mm}$ & $0.97 \mathrm{~mm}$ \\
\hline
\end{tabular}

Table 2 Chemical composition of the specimen in percent

\begin{tabular}{c|c|c|c|c|c|c|c}
\hline $\mathrm{C}$ & $\mathrm{Mn}$ & $\mathrm{Si}$ & $\mathrm{P}$ & $\mathrm{S}$ & $\mathrm{Cu}$ & $\mathrm{Ni}$ & $\mathrm{Cr}$ \\
\hline 0.42 & 0.46 & 0.26 & 0.01 & 0.03 & 0.11 & 0.06 & 0.04 \\
\hline
\end{tabular}

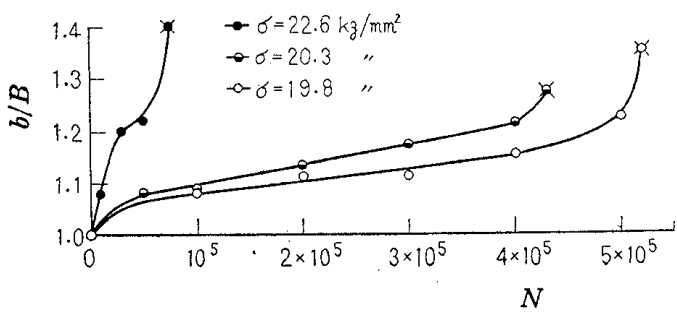

Fig. $6 \quad b / B-N$ curve at a constant stress amplitude for the annealed specimens

\subsection{Measurement of half - value breadth}

$\mathrm{X}$-ray photographs were taken by the method described in the previous papers. The films obtained were examined by an automatic recording microphotometer of the type commonly used in the spectrochemical analysis. Half-value breadths were measured on the curves of diffraction intensity as shown in Fig. 1. The measurement was repeated several times on the films at slightly different positions perpendicular to the scanning direction, and the average values. were taken.

\subsection{Experimental results}

(a) Annealed specimen

Fatigue tests under constant stress amplitudes. were made prior to the tests under varying stress amplitudes in order to obtain a basic knowledge. The changes in half-value breadths in the fatigue process under constant stress amplitudes were obtain-

Table 3 (A) Programs of fatigue tests in the case of ascending or descending stressing for the annealed specimens

\begin{tabular}{c|c|c|c}
\hline \hline$\sigma_{1} \mathrm{~kg} / \mathrm{mm}^{2}$ & $\sigma_{2} \mathrm{~kg} / \mathrm{mm}^{2}$ & $n_{1}$ & $n_{1} / N_{1}$ \\
\hline 19.8 & 20.3 & $8 \times 10^{4}$ & 0.15 \\
19.8 & 20.7 & $8 \times 10^{4}$ & 0.15 \\
19.8 & 22.6 & $8 \times 10^{4}$ & 0.15 \\
19.8 & 20.3 & $4 \times 10^{4}$ & 0.07 \\
19.8 & 20.3 & $2 \times 10^{5}$ & 0.5 \\
\hline
\end{tabular}

\begin{tabular}{c|c|c|c}
\hline \hline$\sigma_{1} \mathrm{~kg} / \mathrm{mm}^{2}$ & $\sigma_{2} \mathrm{~kg} / \mathrm{mm}^{2}$ & $n_{1}$ & $n_{1} / N_{1}$ \\
\hline 22.6 & 21.7 & $3 \times 10^{4}$ & 0.3 \\
22.6 & 20.3 & $3 \times 10^{4}$ & 0.3 \\
22.6 & 20.3 & $10^{4}$ & 0.1 \\
22.6 & 20.3 & $4 \times 10^{4}$ & 0.5 \\
\hline
\end{tabular}

Table 3 (B) Programs of fatigue tests in the case of ascending or descending stressing for the cold worked specimens

\begin{tabular}{c|c|c|c}
\hline$\sigma 1 \mathrm{~kg} / \mathrm{mm}^{2}$ & $\sigma_{2} \mathrm{~kg} / \mathrm{mm}^{2}$ & $n_{1}$ & $n_{1} / N_{1}$ \\
\hline 38.7 & 39.6 & $1.7 \times 10^{5}$ & 0.3 \\
38.7 & 41.5 & $: 1.7 \times 10^{5}$ & 0.3 \\
\hline
\end{tabular}

\begin{tabular}{c|c|c|c}
\hline$\sigma_{1} \mathrm{~kg} / \mathrm{mm}^{2}$ & $\sigma_{2} \mathrm{~kg} / \mathrm{mm}^{2}$ & $n_{1}$ & \multicolumn{1}{c}{$n_{1} / N_{1}$} \\
\hline 47.1 & 42.6 & $5 \times 10^{4}$ & 0.3 \\
42.6 & 41.5 & $1.5 \times 10^{5}$ & 0.3 \\
\hline
\end{tabular}

Table 3 (C) Programs of fatigue tests in the case multiple repeated stressing for the annealed and the cold worked specimens

\begin{tabular}{c|c|c|c|c|c}
\hline$\sigma \alpha \mathrm{kg} / \mathrm{mm}^{2}$ & $\sigma_{2} \mathrm{~kg} / \mathrm{mm}^{2}$ & $n_{1}$ & $n_{1} / N_{1}$ & $n_{2}$ & $n_{2} / N_{2}$ \\
\hline 18.9 & 19.8 & $2.5 \times 10^{5}$ & 0.35 & $1.3 \times 10^{5}$ & 0.3 \\
18.9 & 21.7 & $2.5 \times 10^{5}$ & 0.35 & $5 \times 10^{4}$ & 0.3 \\
\hline \hline$\sigma 1 \mathrm{~kg} / \mathrm{mm}^{2}$ & $\sigma_{2} \mathrm{~kg} / \mathrm{mm}^{2}$ & $n_{1}$ & $n_{1} / N_{1}$ & $n_{2}$ & $n_{2} / N_{2}$ \\
\hline 38.8 & 39.6 & $1.7 \times 10^{5}$ & 0.3 & $1.3 \times 10^{5}$ & 0.3 \\
38.8 & 41.5 & $1.7 \times 10^{5}$ & 0.3 & $10^{5}$ & 0.3 \\
\hline
\end{tabular}


ed as shown in Fig. 6. It is found from this figure that the half-value breadth increases markedly in the early period of stress cycles (the first stage of fatigue), thereafter gradually (the second stage of fatigue), and finally it broadens greatly (the third stage of fatigue). It is interesting to note that the value of $b / B$ just before fracture is nearly constant, irrespective of the magnitude of applied stress.

Fatigue tests under varying stress amplitudes were carried out according to the programs indicated in Table 3 , and observation of the changes in halfvalue breadths was made in the same way as before. Figs. 7 and 8 show the results for the case of ascending stressing. In the case of Fig. 7 stress amplitude was raised after applying the pre-stress during the cycle ratio of $n_{1} / N_{1}=0.15$, and Fig. 8

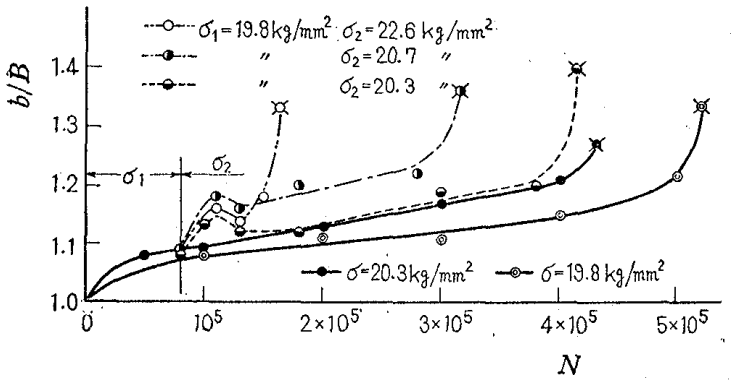

Fig. $7 b / B-N$ curve for the case of ascending stressing (annealed specimen)

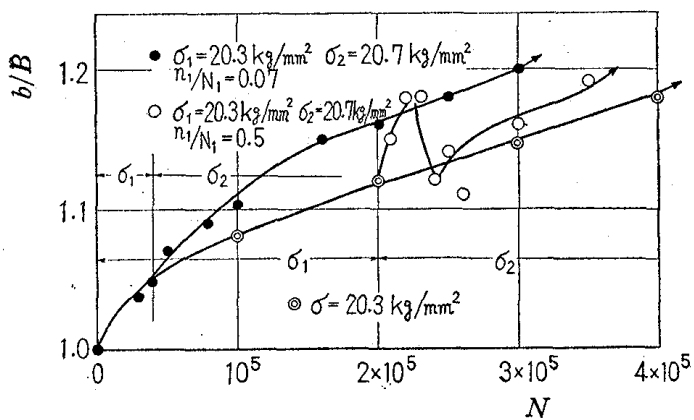

Fig. 8 Influence of pre-stress cycle ratio on the change in half-value breadth for annealed specimen

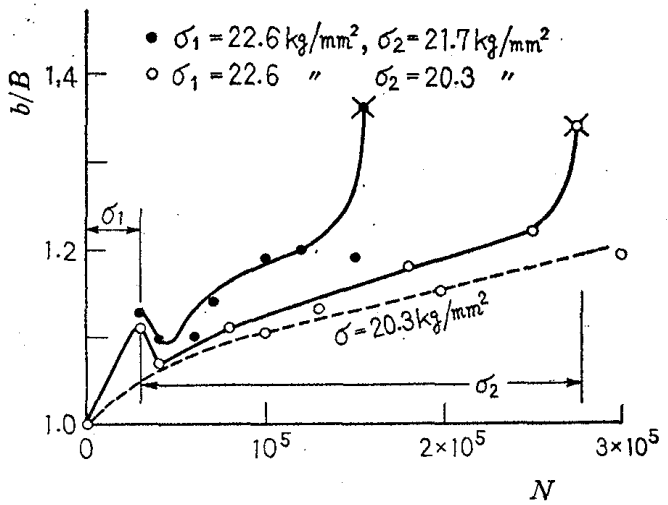

Fig. $9 b / B-N$ curve for the case of descending stressing (annealed specimen) shows the effect of pre-stress cycle ratio on the change in half-value breadth. Looking at Figs. 7 and 8 , it is seen that the number of stress cycles under the pre-stress does not exert a notable influence on the transient increase in half-value breadth after the stress level is raised although it affects the fatigue life considerably. When the stress amplitude is raised at the cycle ratio of $n_{1} / N_{1}=0.5$ or 0.15 to the secondary stress level, the half-value breadth increases markedly in the early period of the second. ary stress cycles, then decreases with the number of cycles, and finally comes close to the value of $b / B$ under a constant stress amplitude at the secondary stress level. In the case of $n_{1} / N_{1}=0.07$, however, such transient increase in half-value breadth does not appear. It should be emphasized here that in these cases also, the value of $b / B$ just before fracture is nearly constant.

A series of tests were run for the case of descending stressing, and the $b / B$ vs. $N$ relations obtained are shown in Figs. 9 and 10. In the figures, a rapid reduction in the value of $b / B$ is observed at the transition point of applied stress. However, general features of these curves are quite similar to those for the case of constant stress amplitudes.

The fatigue tests under multiple repeated stress according to the programs shown in Table $3(\mathrm{C})$ were

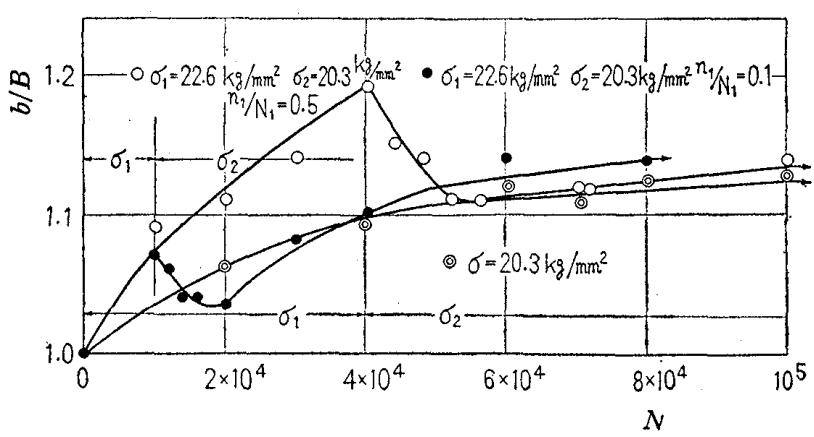

Fig.10 Influence of pre-stress cycle ratio in the case of descending stressing on the change in half-value breadth (annealed specimen)

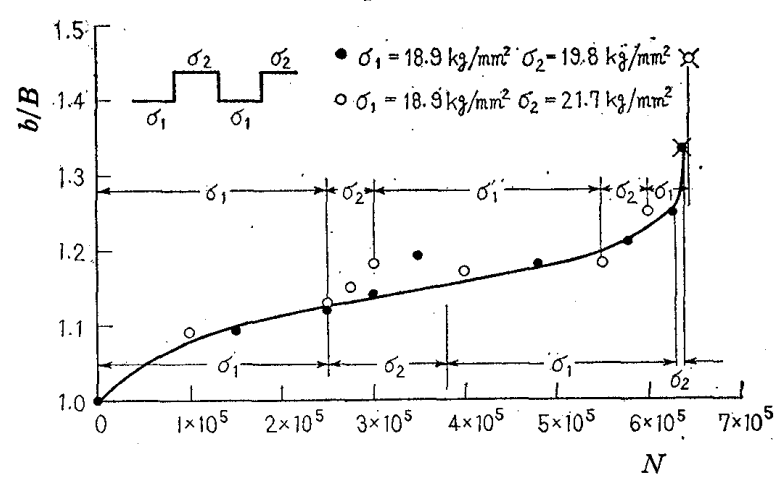

Fig.11 $b / B-N$ curve for the case of multiple repeated stressing (annealed specimen) 
carried out for the annealed specimens, and the change in half-value breadth was observed. The $b / B-N$ relation obtained is shown in Fig. 11 . From this figure, it is seen that the transient increase or decrease in the value of $b / B$ is obtained successively at the transition point of stress level, but general aspect of the curve is nearly the same as that under a constant stress amplitude.

\section{(b) Cold worked specimen}

A similar study was made on the cold worked material. The trend of the change in half-value breadth for this case is dissimilar to that for the case of the annealed material, and a steady decrease in half-value breadth occurs with the progress of stress cycles. Results of the tests are shown in Figs. 12 and 13. Figs. 12 and 13 are for the case of ascending and descending stressing, respectively. The value of half-value breadth decreases noticeably in the early period of stress cycles, and thereafter fades gradually. As is apparently from these figures, similarly to the case of the annealed material, any notable increase or decrease in half-value breadth at the transition point of stress amplitude is not observed. The important fact which should be mentioned here is that the value of $b / B$ just before fracture is constant, irrespective of the magnitude of applied stress and its history. Fig. 14 shows the relation between $b / B$ and $N$ in the case of multiple repeated stressing. In the figure, it is observed that the $b / B \cdot N$ curve for this case coincides with that under a constant stress amplitude.

\section{Discussion of the results}

A brief discussion on the change in half-value breadth under a constant stress amplitude has bsen made with relation to the mechanism of fatigue failure. In an annealed state, the material is free from internal stress and damage. When the annealed specimens are subjected to repeated stress, work hardening ${ }^{(8)}$ occurs in certain crystal grains, as a result of plastic deformations in special directions of atomic planes in an early stage of stress repetitions. The steep increase in half-value breadth in the first stage of fatigue is caused by these slip-phenomena. In the course of the second stage of fatigue, nucleation and growth of fatigue damage proceed. The authors assume slip hand cracks that would be created in certain crystal grains of the material as a result of the crystal slips already produced. The half-value breadth in the second stage of fatigue involves both the influences of micro-stress around micro-cracks and of micro-cracks themselves.

In the case of ascending stressing, it is considered that the stepwise increase of primary stress level to the secondary one would further accelerate crystal

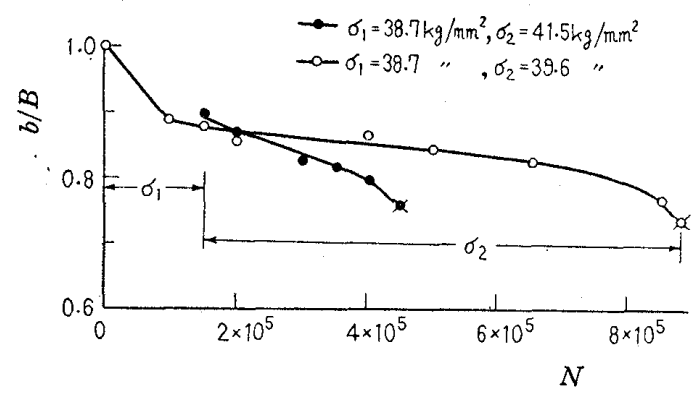

Fig.12 $b / B-N$ curve for the case of ascending stressing (cold worked specimen)

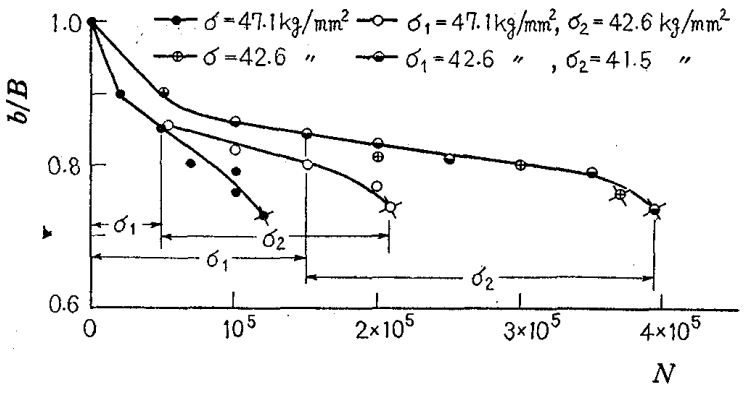

Fig.13 $b / B-N$ curve for the case of descending stressing (cold worked specimen)

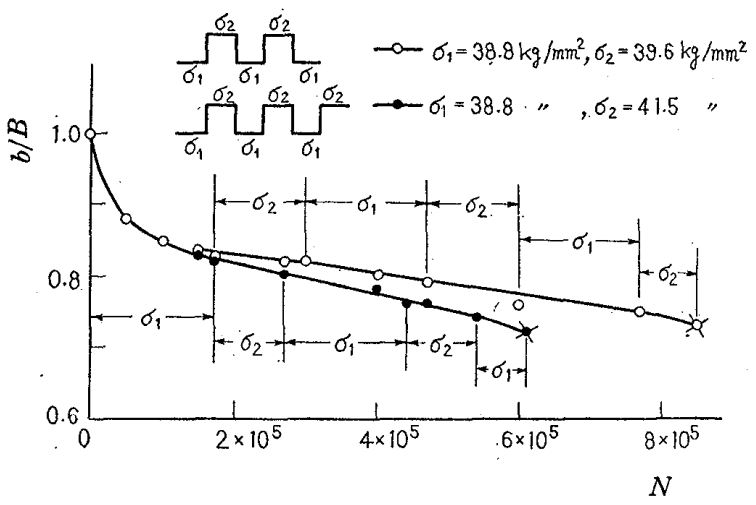

Fig.14 $b / B-N$ curve for the case of multiple repeated stressing (cold worked specimen)

slips along certain crystal planes in favourably. oriented grains. This would cause a continuous increase in half-value breadth, and the slope of $b / B-N$ curve for this case comes close to that under a constant secondary stress amplitude. When the stress amplitude is raised during the secondary stage of fatigue, it is assumed that the rise of stress level would induce slips in random directions around the micro-cracks which have been produced already in crystal grains during the pre-stress cycles. This would cause a considerable increase in half-value breadth, but the random micro-stresses thus induced would be released easily by further repetition of stresses, since these fatigue slips proceed in original slip planes. Moreover, it is supposed that the appearance of micro-cracks causes the relaxation of 
randcm micro-stresses. In this way, the $b / B-N$ curve rises showing a temporary increase at the transition point of stress level, and then falls like the curve under the initially secondary stress amplitude.

In damaged materials, micro-stress concentrations are prcduced by repeated stress, which would supply the energy for the growth of micro-cracks. On the other hand, in the case of descending stressing, accumulated micro-stress would be relaxed to a certain value corresponding to that under the secondary stress.

In the case of cold worked specimens, work hardening, fragmentation, and disorientation are produced simultaneously in the crystal grains. The micro-stresses in random directions would be in a state of high potentiality. Therefore, in the process of applying alternating stress to initially cold worked specimens, relaxation of micro-stresses would be expected in specific directions of certain grains. Along the specific directions of these grains specified by the direction of alternating stress, micro-strains would accumulate in the crystals as a result of plastic deformations, similarly to the case of the annealed materials. Thus, in the prccess of applying repeated stress to cold worked specimens, two opposite sorts of phenomena prcceed simultaneously. Under varying stress amplitude, the influence of the relaxation of random micro-stresses on the change in half-value breadth is predominant over that of the accumulation of micro-stresses in specific directions. The observed trend of the change in half-value breadth during fatigue under varying stress amplitudes could be understood in this way.

\section{Prediction of fatigue life}

In the previous papers ${ }^{(1)(2)}$, it was reported that the possibility of predicticn of fatigue damage of materials subjected to cyclic stressing under a constant stress amplitude lies in the linear relation of $b / B$ to $\log n / N$. However, it is questionable whether this relation would hold even for the case of fatigue under varying stress amplitudes. This problem will be examined in the light of the results of present experiments.

According to the Miners concept of cumulative fatigue damage, fatigue failure would occur when the summation of the cycle ratios under various stress levels becomes unity. The authors considered that the change in half-value breadth due to stress repetitions would correspond to the fatigue damage. The authors tried to reveal the relation between the change in half-value breadth and the summation of cycle ratios. The relation of $b / B$ to the $\log$ of the summation of cycle ratios $\log \sum n / N$ for the case of Fig. 11 is indicated in Fig. 15. The value of $b / B$ increases

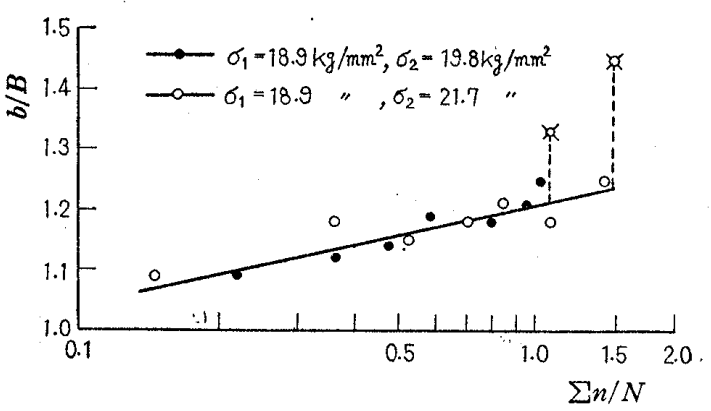

Fig.15 $b / B-\log \left(\sum n / N\right)$ curve for the annealed specimens

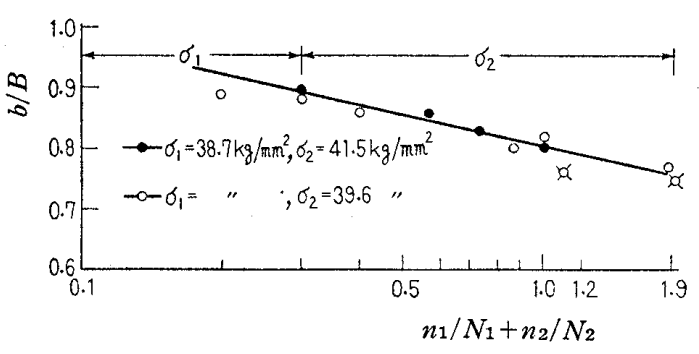

Fig.16 $b / B-\log \left(n_{1} / N_{1}+n_{2} / N_{2}\right)$ curve for the cold worked specimens

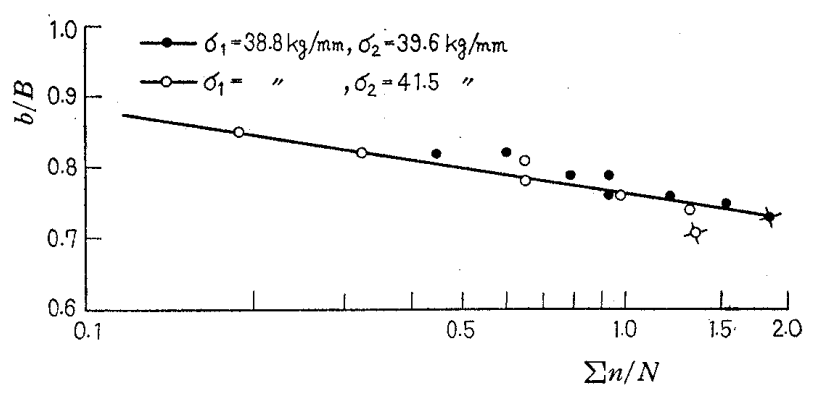

Fig.17 $b / B \cdot \log \sum n / N$ curve for the cold worked specimens

linearly with the value of $\log \sum n / N$, irrespective of the magnitude of the secondary stress. Fig. 16 shows the case of ascending stressing for the cold worked specimens. The $b / B-\log \left(n_{1} / N_{1}+n_{2} / N_{2}\right)$ curve decreases linearly until just before fracture. In the case of multiple repeated stressing, the relation of the change in half-value breadth to the log of summation of cycle ratios $\log \sum n / N$ is similar to the curve of Fig. 16, and is shown in Fig. 17. The relation between $b / B$ and $\log n / N$ is represented by an ascending (Fig. 15) or a descending (Fig. 16 and 17) straight line for each material tested, irrespective of the magnitude of the secondary stress, just as in the case of the previous results. As mentioned above, the prediction of fatigue life by means of X-ray would be possible for the case of ascending, descending and multiple repeated stressing just in the same way as reported previously.

\section{References}

(1) S. Taira and K. Honda : Bulletin of JSME, Vol.4, 
No.14, (1961), p.230.

(2) S. Taira and K. Honda : Trans. Japan Inst. Metals, Vol.1 (1960), p.43.

(3) W.A. Rachinger: Jour. Sci. Instrum., Vol. 25 (1948), p. 257.

(4) L.G. Finch : Nature, Vol.163 (1946), p.402.

(5) H. Neerfeld : Mitt-K.Wilh-Inst. Eisenforsch., Bd.
27 (1944), S. 16

(6) F.W. Jones: Proc. Roy. Soc. Lond., Ser. A, Vol. 166 (1938), p.16.

(7) C.G. Shull : Phys, Rev., Vol.76 (1946), p.679.

(8) A.H. Cottrell : Dislocation and Plastic Flow in Crystals, (1953), Oxford.

539. 389. 3:669. 15'26'24-194

\title{
Relaxation of $2^{1 / 4}$ Cr-1 Mo Steel*
}

\author{
By Shuji TAIRA**, Fusayuki Suzuki***, \\ and Masaru YAMAGUCHI****
}

\begin{abstract}
Relaxation, step down and creep tests were carried out on a $\mathrm{Cr}$-Mo steel at a temperature of $500^{\circ} \mathrm{C}$ and the characteristics of the material obtained by these tests are discussed in order to correlate them to each other. It was found that the log-log plot of stress versus strain rate in relaxation lies on a broken straight line. It may be considered that the relaxation in the higher stress range corresponds to transient creep and that in the lower stress range to steady creep. This simple correspondence does not always hold, because the stress decrease during relaxation is accompanied by creep recovery. The predictions of relaxation using the informations obtained from other sorts of tests, which have been proposed up to this time, are not always satisfactory due to the lack of consideration of relaxation characteristics. Using the method presented by the authors, which is based on the fundamental relation between stress and plastic strain rate, the residual stress can be satisfactorily predicted. It is also found that a step down test is effective for determining the relaxation properties of materials.
\end{abstract}

\section{Introduction}

Creep test is generally adopted as a basic test for studying the fundamental strength characteristics of metals at elevated temperatures and the greatest interest of the investigators in this field is aroused on creep problems. However, there are many sorts of structural members which transmit a force through them, and the creep in these stressed members may cause a gradual decrease in the stress initially applied. In designing these components, the relaxation property of materials instead of creep should be taken into account. In practice, it is also desirable to predict relaxation from creep data available. Almost all of the studies ${ }^{(1)-(6)}$ on the relaxation of metallic materials which have been reported up to date aimed at obtaining the relaxation data of individual materials. Some of these papers are concerned with the prediction of relaxation from creep data, but it

* Received 29 th July, 1961.

** Professor, Faculty of Engineering, Kyoto University.

*** Graduate Student, Faculty of Engineering, Kyoto University, Sakyo-ku, Kyoto.

**** Member, Kawasaki Iron and Steel Mfg. Co. is not likely that satisfactory prediction can be made from these papers.

In this connection, as had been reported in the previous paper( ${ }^{(7)}$, the authors carried out a series of experiments using a low carbon steel to obtain the relaxation and the creep characteristics of the material in order to elucidate the relation of these charact:ristics. As the result, it was found that in a relaxation test the linear relation of $\log$ plastic strain rate to log stress was held and it was the same as the relation obtained from the step down test. It was also proved that this linear relation was approximately predicted from creep data. However, these results were confined within the range of transient creep and short time relaxation because of the short test duration.

In the present study, experiments were carried out on a $2^{1 / 4} \mathrm{Cr}-1$ Mo steel with the aim of knowing whether the linear relation of $\log$ strain rate to $\log$ stress holds after a long time relaxation as well as in a short time test. It was also intended to find the relationship between relaxation and creep, and to discuss the prediction of relaxation from other sorts 